\title{
The Synthesis and Characterization of h-BN Nanosheets with High Yield and Crystallinity
}

\author{
Shaocheng Li, Xianlang Lu, Yanda Lou, Kejun Liu, and Benxue Zou* \\ Cite This: ACS Omega 2021, 6, 27814-27822 \\ Read Online
}

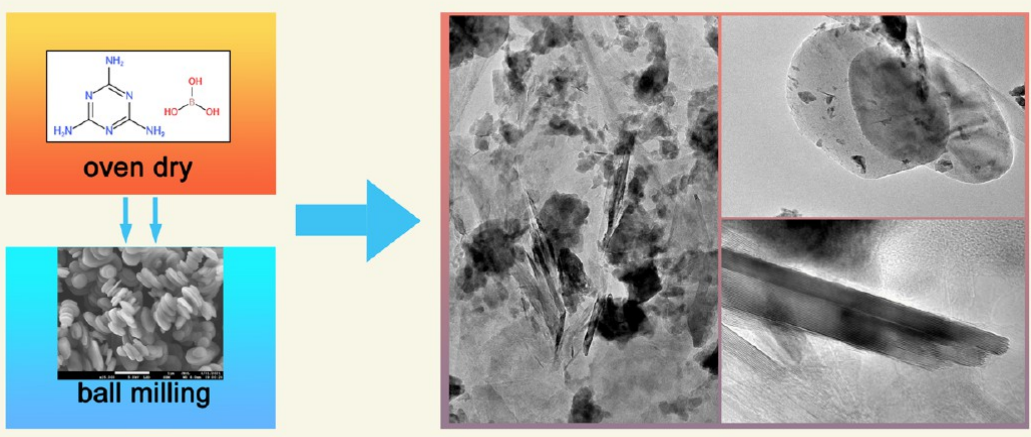

ABSTRACT: Nowadays, boron nitride (BN) has attracted a great deal of attention due to its physical and chemical properties, such as high-temperature resistance, oxidation resistance, heat conduction, electrical insulation, and neutron absorption. The unique lamellar, reticular, and tubular morphologies and physicochemical properties of BN make it attractive in the fields of adsorption, catalysis, hydrogen storage, thermal conduction, insulation, dielectric substrate of electronic devices, radiation protection, polymer composites, medicine, etc. Based on this, we propose a novel method to produce boron nitride nanosheets (BNNSs) by a two-step method. The structure and morphology of the prepared BNNSs were characterized by scanning electron microscopy, transmission electron microscopy, atomic force microscopy, XRD, FTIR, etc. The results showed that the prepared BNNSs had high crystallinity and the stripping efficiency of h-BN as well as the performance and yield of BNNSs had been improved, and the cost and environmental pollution of BNNS preparation had been reduced accordingly.

\section{INTRODUCTION}

Hexagonal boron nitride ( $\mathrm{h}-\mathrm{BN})$ is known by the name white graphite. ${ }^{1-3}$ As an isoelectronic body of graphite, h-BN that possesses a graphene-like two-dimensional layered structure exhibits excellent insulation, ${ }^{4}$ thermal conductivity, ${ }^{5}$ and thermal stability. 6,7 High-temperature resistance, pressure resistance, chemical corrosion resistance, anti-oxidation property, and good neutron radiation shielding performance make $h$ BN a new material with excellent performances and great development potential. ${ }^{8}$ The h-BN in-plane $\mathrm{B}$ and $\mathrm{N}$ atoms are associated by $\sigma$ covalent bonds, forming a honeycomb-like structure with an interlayer thermal conductivity that can reach $300 \mathrm{~W} /(\mathrm{m} \cdot \mathrm{K})$ and still maintain its properties at $900{ }^{\circ} \mathrm{C}$. ${ }^{9}$ Due to different $\mathrm{p}$ orbital energies of $\mathrm{B}$ and $\mathrm{N}$ atoms, the symmetry of the sublattice is destroyed, leaving $\mathrm{h}-\mathrm{BN}$ with a high band gap width $(5-6 \mathrm{eV})$ and the ability to be an ideal insulating material. ${ }^{10}$ A conventional h-BN crystal has large particle size, low specific surface area, natural tolerance to other chemicals, and lack of surface level molecular binding sites, which makes it difficult for h-BN to interface with other materials. ${ }^{11}$ By deeply functionalizing and nanosizing h-BN, it will not only improve the specific surface area active sites of $h-B N$ but also increase the compatibility of h-BN with other molecules, ${ }^{12}$ which will open the applications of h-BN materials in many other emerging fields, such as aerospace, ${ }^{13}$ biomedicine, ${ }^{14}$ electronics, ${ }^{15}$ hydrogen storage, ${ }^{16}$ and so on. The following is a detailed description of some of its applications.

Talmon et al., 8 researchers from the University of Illinois, separated the boron nitride layer into atomically thick sheets with a super acid treatment and formed binding sites on the surface of the boron nitride atomically thick sheets, providing a two-dimensional interface with nanoparticles, molecules, and other devices for optoelectronic and piezoelectric devices, passivation layers for solar cells, medical diagnostic devices, and many other applications. Li et al. ${ }^{17}$ prepared monolayer boron nitride with a larger area by the vapor deposition method and

Received: June 29, 2021

Accepted: September 24, 2021

Published: October 15, 2021 
reported for the first time that electric charge movement and transport can be performed in the plane of monolayer boron nitride, similar to organic semiconductor properties. Lu et al. ${ }^{18}$ exfoliated h-BN powder in thionyl chloride to produce BNNSs without using dispersion agents. Through using the depositionprecipitation method, Pd nanoparticles were immobilized onto BNNS surfaces to construct a Pd-BNNS catalyst that exhibited high catalytic activity and recyclability for the hydrogenation of nitro aromatics. In addition to this, $\mathrm{Lu}$ and his team also used molten citric acid to treat $\mathrm{h}-\mathrm{BN}$ in order to produce aqueous dispersible boron nitride sheets. ${ }^{19}$ Then, they used said BNNSs to fabricate hydrogels with high water retentivity and flexibility, revealing the application prospect of BNNSs as a highperformance hydrogel. Miele and his team have proposed a new strategy for the production of PVA-based porous membranes using mainly water as solvent and hexagonal boron nitride nanosheets (BNNSs) as a stabilizer. The results show that BNNSs/PVA porous membranes fabricated by the Pickering emulsion template method are good candidates for use as microfiltration membranes. ${ }^{20}$ In addition to this, Miele and his team have reported a simple, effective, and green way for the fabrication of gelatin-graphene-like BN nanocomposites for gas barrier applications. The reinforcement effect of graphenelike $\mathrm{BN}$ on the gelatin properties is discussed. The obtained graphene-like BN nanocomposites show good dispersion in the gelatin matrix and remarkable capability to improve the crystallinity and the barrier properties of gelatin. The barrier properties of gelatin/BN nanocomposites have been enhanced by a factor of 500 at 2 bar compared to a gelatin film without graphene-like $\mathrm{BN}^{21}$

While hexagonal boron nitride nanosheets (BNNSs) have thermal and mechanical properties similar to graphene, ${ }^{22}$ they also possess cathodic luminescence, ${ }^{23}$ electrical insulation, ${ }^{24}$ high thermal conductivity, ${ }^{24}$ thermal stability, ${ }^{25}$ and hightemperature antioxidant properties. ${ }^{26}$ BNNSs can also be used as polymer additives, ${ }^{27}$ carriers for nanoparticles, ${ }^{28}$ highly durable field emitters, ${ }^{29}$ superhydrophobic films, ${ }^{30}$ etc., making BNNSs have a huge application market and promise. ${ }^{31-33}$ Miele and his team have given a very detailed overview of synthetic methods for the production of boron nitride nanocomposites, especially polymer- and ceramic-based nanocomposites, and their potential applications in the fields of energy, environment, and health. ${ }^{34}$ This work has served as a great inspiration for the direction of future experiments.

Nevertheless, compared with graphene, h-BN shows stronger interlayer van der Waals force due to the existence of ionic bonds between adjacent layers, making boron nitride harder to intercalate and peel than graphene. ${ }^{35}$ Current methods for the preparation of BNNSs mainly include a "bottom-up" vapor deposition method ${ }^{36}$ and chemical exfoliation ${ }^{37}$ as well as "topdown" ultrasound-assisted solvent exfoliation ${ }^{38}$ and mechanical exfoliation method. ${ }^{39}$ Among them, the chemical vapor deposition (CVD) method, in which h-BN coating materials are obtained by reactive deposition using gaseous precursor species containing boron and nitrogen elements on the substrate surface, has a wide adjusting range of parameters, welldistributed and compact coated products, higher purity, and better thermal, force, electrical, and other properties compared with other methods. ${ }^{36}$ However, CVD preparation methods require extremely high temperatures, up to $900-1300{ }^{\circ} \mathrm{C}$, as well as high equipment costs and low yield, making it difficult for large-scale production. ${ }^{40}$ The chemical exfoliation method, which draws on the chemical oxidation method to prepare graphene to achieve effective exfoliation, uses concentrated $\mathrm{H}_{2} \mathrm{SO}_{4}, \mathrm{KMnO}_{4}$, and $\mathrm{H}_{2} \mathrm{O}_{2}$ to chemically exfoliate h-BN. ${ }^{37}$ Although BNNSs can be obtained by this method, the yield is also low and the starting materials are neither green nor environmentally friendly. ${ }^{41}$ The mechanical ball milling method utilizes hard balls to peel bulk h-BN into a few layers or a single layer of boron nitride nanosheets under the action of high shear and impact forces. ${ }^{33}$ It has been proven that short ball milling time results in low stripping efficiency, while excessive ball milling time damages the BN structure, leading to severely decreased surface crystallinity and more crystal defects. ${ }^{42}$ In addition to the above methods, a green approach to prepare exfoliated hexagonal boron nitride nanosheets (BNNSs) has been presented by Miele and his team. The first step involves the dispersion of pristine $\mathrm{h}-\mathrm{BN}$ within an aqueous solution containing gelatin and potassium or zinc chloride using a sonication method. The second step involves the removal of larger exfoliated BNNSs through a centrifugation procedure. ${ }^{43}$ The success of this experiment makes it possible to improve the yield of exfoliated BNNSs by controlling the different factors that may affect exfoliation. It also served as an irreplaceable inspiration for the design of this experiment.

Recently, in collaboration with Liaoning Dandong Institute of Chemical Research Co., Ltd., China, our college carried out the research work on functionalization and nanosizing of boron nitride, including surface modification of boron nitride, development of a boron-carbon-nitrogen composite, etc. The obtained boron-carbon-nitrogen composite exhibits an obvious $2 \mathrm{D}$ intercalation structure of boron nitride and graphene, which effectively reduces the band gap width of boron nitride and exhibits certain semiconductor properties and fine electrochemical sensing performance. ${ }^{44}$ In this study, submicrometersized h-BN was first synthesized via a high-temperature solidphase method and the effects of different reaction media on the structure and morphology of h-BN were investigated. Then, the submicrometer-sized h-BN was used as a ball milling raw material to effectively exfoliate it by the mechanical ball milling method. The effects of factors such as additives on the structure and morphology of BNNSs were also investigated. The results of this study showed that the BNNSs with uniform size distribution, which possess advantages of high yield, simple postprocessing, and less pollution, can be obtained by appropriately adjusting factors such as the ball-to-material ratio and ball milling additives. To the best of our knowledge, this is the first time that a novel two-step method to produce boron nitride nanosheets (BNNSs) is reported.

\section{EXPERIMENTAL SECTION}

2.1. Materials. Boric acid was purchased from Bor Mining Cooperation (Russia) with a purity of $99.99 \%$, and the CAS number is 10043-35-3. Melamine was provided by Shandong Helitai Chemical Co., Ltd. (China) with a purity of $99 \%$, and the CAS number is 108-78-1. Sodium fluoride (99\%, 7681-49-4), potassium chloride (99\%, 7447-40-7), calcium chloride (99\%, 10035-04-8), and sodium chloride (99.5\%, 7647-14-5) were obtained from Sigma-Aldrich.

2.2. Preparation of Submicrometer-Sized h-BN. Melamine, sodium fluoride, and boric acid were weighed and mixed in a ratio of $1: 5: 17.5$, and then the mixture was pressed into sheets before being oven dried at a constant temperature of $250{ }^{\circ} \mathrm{C}$ for $12 \mathrm{~h}$. The dried material was placed into a medium frequency induction furnace where the temperature was raised to $1150{ }^{\circ} \mathrm{C}$ at a rate of $400{ }^{\circ} \mathrm{C} / \mathrm{h}$ and baked for $8 \mathrm{~h}$ under 


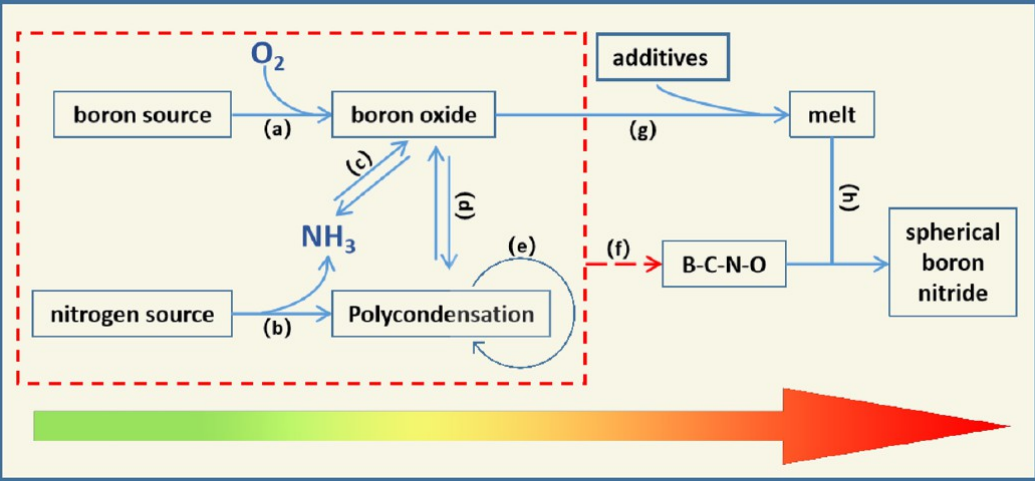

Figure 1. Flow chart of the reaction of solid boron-nitrogen sources after heating.

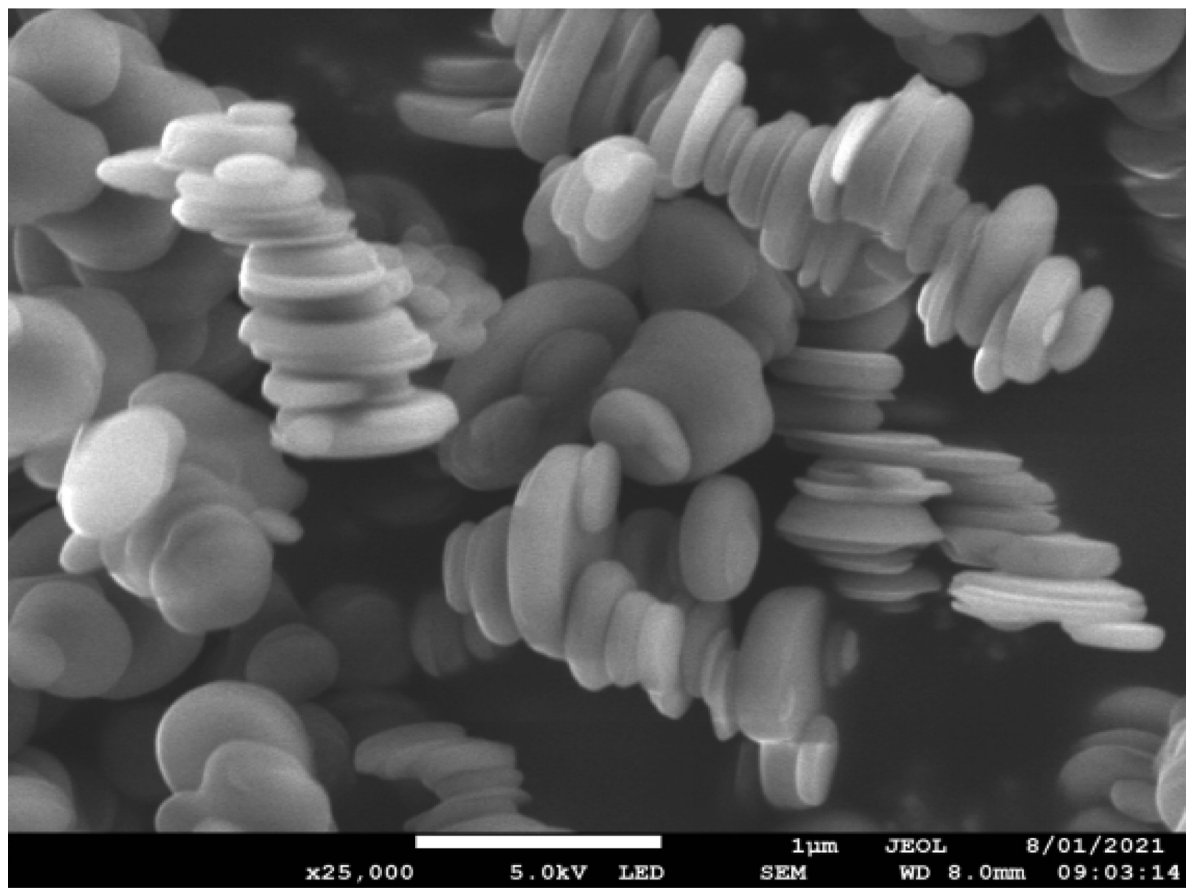

Figure 2. SEM images of submicrometer h-BN.

nitrogen protection for a high-temperature solid-phase reaction. After crushing, washing, pickling, filtering, and drying, submicrometer h-BN was obtained. According to the same procedure, h-BN was prepared by a high-temperature solid-state reaction with different molten salts, including potassium chloride, calcium chloride, and sodium chloride, to study how different molten salts would affect the structure and properties of h-BN.

2.3. Preparation of h-BN Nanosheets. The submicrometer-scale h-BN and water were added into a planetary ball mill at a certain ratio by adding different sizes and ratios of zirconia balls and adjusting the planetary rotation speed and $z$-axis rotation speed with forward-reverse alternating operation, to perform the ball milling process. The sample was retrieved after ball milling was done. After that, the zirconia balls were washed with water to collect the washing solution that was then carried out by vacuum filtration with a microporous membrane with a pore size of $0.22 \mu \mathrm{m}$. The sample was washed several times with distilled water and then dispersed into distilled water or ethanol to obtain BNNS dispersions, which then went through vacuum drying to acquire BNNS powder. Determination of the

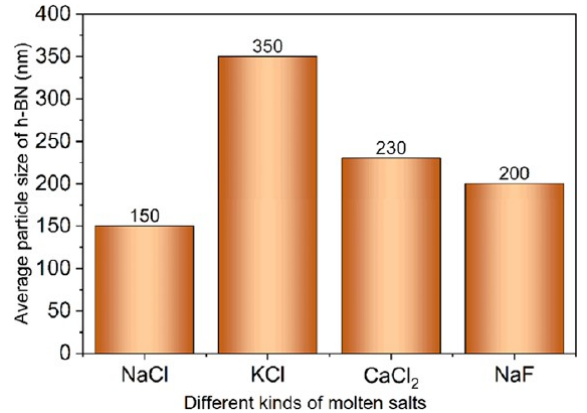

Figure 3. Average particle size of h-BN synthesized with different molten salts.

concentration of the BNNS dispersion was as follows: After standing for a period of time, a certain volume of the upper liquid was taken out from the obtained BNNS dispersions with a pipette gun and transferred to a clean vial that had been weighed. Then, the vial was placed in a drying oven to dry at $90{ }^{\circ} \mathrm{C}$ for $24 \mathrm{~h}$ to evaporate the solvent. The total mass of the vial and the dry 


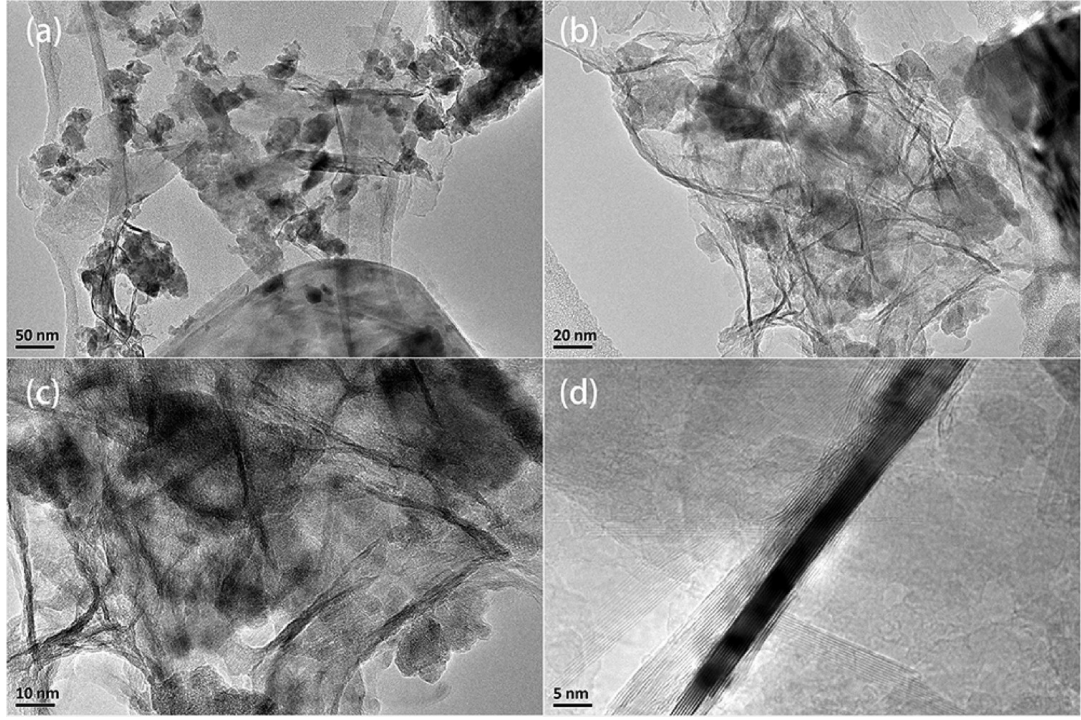

Figure 4. TEM images of BNNs obtained from submicrometer-scale h-BN after ball milling under magnifications of (a) 5000 times, (b) 12,500 times, (c) 25,000 times, and (d) 50,000 times.

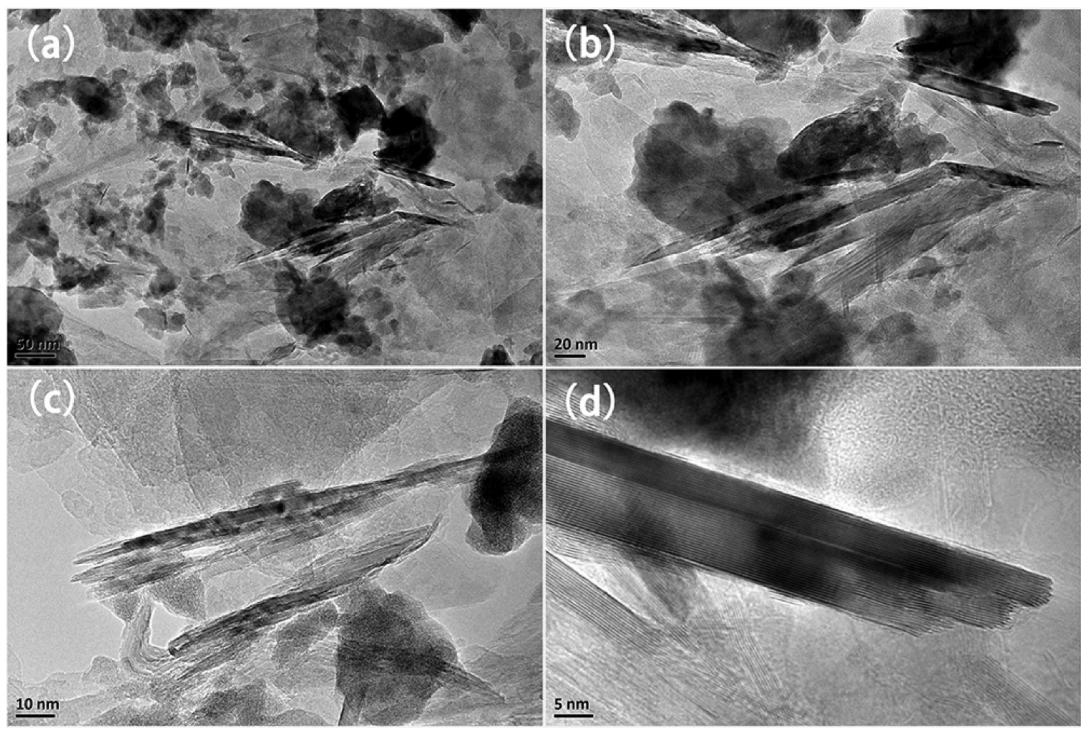

Figure 5. TEM images of BNNSs obtained from commercially available h-BN (23 $\mu \mathrm{m}$ D50) after ball milling under magnifications of (a) 5000 times, (b) 12,500 times, (c) 25,000 times, and (d) 50,000 times.

solid was weighed, and the concentration of nanosheets was calculated by the subtractive method, as shown below.

$$
C=\frac{M-m}{V}
$$

where $C$ stands for the concentration of boron nitride nanosheet dispersion $(\mathrm{mg} / \mathrm{mL}), M$ stands for total mass of the vial and solid dry matter, $m$ stands for the mass of vial $(\mathrm{mg})$, and $V$ stands for the volume of the removed upper liquid $(\mathrm{mL})$.

2.4. Characterization. Morphologies of the samples were investigated by field emission scanning electron microscopy (SEM, FEI, Quanta FEG250, USA) and transmission electron microscopy (TEM, FEI, Tecnai G2 F20, USA). The Fourier translation infrared spectra were recorded on an FT-IR spectrometer (FT-IR, PerkinElmer, Spectrum Two, USA), using the $\mathrm{KBr}$ pelleting method. The crystalline phases of the samples were determined by an X-ray diffractometer (XRD, PANalytical B.V., Thermo Scientific, Netherlands). Atomic force microscopy (AFM) analysis was performed by employing an atomic force microscope (AFM, Multimode 8 Nanoscope V system, USA). EDS measurement was performed using a Zeiss EVO ED15 microscope coupled with an Oxford X-MaxN EDS detector. Surface compositions of BNNSs were observed by Xray photoelectron spectroscopy (XPS, Kratos Axis Ultra DLD). The Raman spectra were obtained from a Horiba xplora. The yield of BNNSs was calculated using the following equation:

$$
\text { yield }(\%)=(\text { weight }(\mathrm{BNNS}) / \text { weight }(\mathrm{h}-\mathrm{BN})) \times 100
$$

\section{RESULTS AND DISCUSSION}

3.1. Characterization of Submicrometer h-BN. 3.1.1. SEM of Submicrometer $h-B N$. Based on the complex heterogeneous reaction of solid boron-nitrogen sources after heating, the following physical and chemical changes occur in turn with the increase of temperature, as shown in Figure 1: (a) 
formation of amorphous boron oxide from the boron source, (b) deamination polycondensation occurred on the nitrogen source, (c) the reaction of newly formed ammonia with boroxides, (d) reaction of boroxides with polycondensation products, (e) ringopening pyrolysis of polycondensation products, (f) formation of amorphous boron-carbon-nitrogen-oxygen compounds, $(\mathrm{g})$ melting of boroxides and reaction additives, and $(\mathrm{h})$ formation of spherical boron nitride by decarburization and deoxidation of boron-carbon-nitrogen-oxygen compounds in melt. Figure 2 shows the scanning electron micrograph of submicrometer-sized h-BN obtained after the high-temperature solid-phase reaction at $1200^{\circ} \mathrm{C}$ for $8 \mathrm{~h}$, from which it can be seen that the particle size of h-BN is submicrometer sized. The particle size of h-BN was between 200 and $500 \mathrm{~nm}$ and evenly distributed.

3.1.2. Effects of Molten Salts on Submicrometer $H-B N$. Solid-phase reactions in which a certain amount of reaction media, such as alkali molten salts $\left(\mathrm{NaF}, \mathrm{CaF}_{2}, \mathrm{NaCl}, \mathrm{KCl}\right.$, etc.) or alkaline earth metal halides $\left(\mathrm{CaCl}_{2}\right.$, etc. $)$, was added to the solution have an effect both on the formation of submicrometer $\mathrm{h}-\mathrm{BN}$ and the particle size. The addition of molten salt reduces the temperature required for the solid-phase reaction, thus reducing the particle size of the product. Figure 3 shows average particle scales of h-BN synthesized with different molten salts, from which it can be seen that well-dispersed submicrometer globular particles could be obtained from all four kinds of molten salts, in which the particles of $\mathrm{h}-\mathrm{BN}$ obtained by sodium salt had a smaller average size. However, the addition of molten salt may cause some corrosion on the high-temperature reaction furnace.

In addition, factors such as the ratio of raw materials and heating rate have a certain effect on the synthesis of submicrometer h-BN. The amount of fumes at roasting and the subsequent processing volume will increase, and boronic acid feedstock will be wasted if the mass ratio of the nitrogen source and boronic acid is too low. In contrast, an excessive ratio would lead to the failure to form submicrometer boron nitride. The reaction temperature should be controlled between 900 and $1200{ }^{\circ} \mathrm{C}$, below which it will lead to an extremely slow reaction. Above this temperature range, it will lead to the boron nitride crystals' overgrowth to a micrometer scale; hence, it more easily acquires agglomerated products with irregular shape, which will lead to the formation of products with larger particles. The heat holding time is 2 to $4 \mathrm{~h}$; a shorter time leads to an incomplete deoxygenation reaction, while a longer holding time causes boron nitride particles to grow larger.

3.2. Characterization of BNNSs. 3.2.1. TEM of BNNSs. Figure 4 shows the transmission electron micrographs (TEM) of BNNSs obtained after the mechanical ball milling process of submicrometer h-BN under different magnifications. As shown in Figure 4, the obtained BNNSs are transparent and well distributed, exhibiting a wrinkled structure with graphene-like edges with a small amount of agglomeration. The thickness of BNNSs is about 4 to $10 \mathrm{~nm}$, while the number of BNNS layers ranges from about 5 to 15 . This indicates that after mechanical ball milling, h-BN was effectively exfoliated. The exfoliated BNNSs could be well dispersed in aqueous solution to form a homogeneous dispersion with an overall creamy white color. The obtained dispersions appear to be homogeneous and stable with concentrations up to $100 \mathrm{mg} / \mathrm{mL}$.

Figure 5 consists of TEM photographs of BNNSs obtained by mechanical ball milling of commercially available h-BN $(23 \mu \mathrm{m}$ D50) with a large average size of $23 \mu \mathrm{m}$. As shown in Figure 5, the obtained BNNSs are heterogeneous in thickness with low transparency of the sheet-like structure of h-BN. It does not exhibit an obvious graphene-like puckered structure and has more edge defects, the thickness of which is about 7 to $25 \mathrm{~nm}$ and the number of BNNS layers ranges from about 10 to 25 .

3.2.2. XRD Patterns of BNNSs. Figure 6 shows the XRD spectra of submicrometer h-BN and commercially available h-

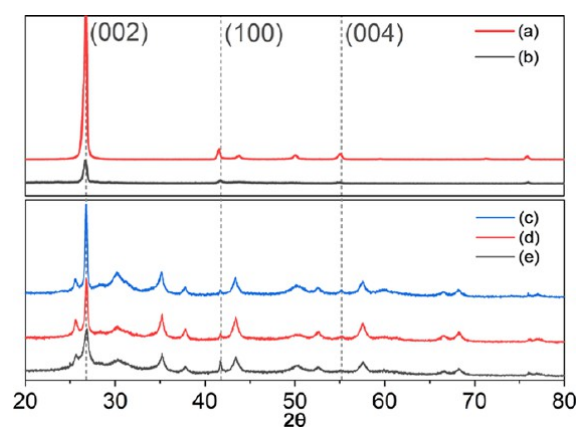

Figure 6. XRD spectra of (a) submicrometer h-BN, (b) commercially available h-BN (23 $\mu \mathrm{m}$ D50), (c) BNNSs obtained from submicrometer h-BN with additives, (d) BNNSs obtained from submicrometer h-BN, and (e) BNNSs obtained from commercially available h-BN (23 $\mu \mathrm{m}$ D50).

$\mathrm{BN}$ before and after ball milling exfoliation, which all have typical $26.5,41.5$, and $55.1^{\circ}$ diffractions of h-BN, corresponding to the (002), (100), and (004) facet diffraction peaks of h-BN, respectively. As can be seen from Figure $6 c-e$, the crystallinity of BNNSs after exfoliation was fairly good. After comparing (a) and (b) with (c), (d), and (e), it can been seen that the crystal system of the samples did not change by ball milling.

3.2.3. FTIR Spectroscopy of BNNSs. By comparing the five FTIR spectra in Figure 7, it can be seen that the peaks before and

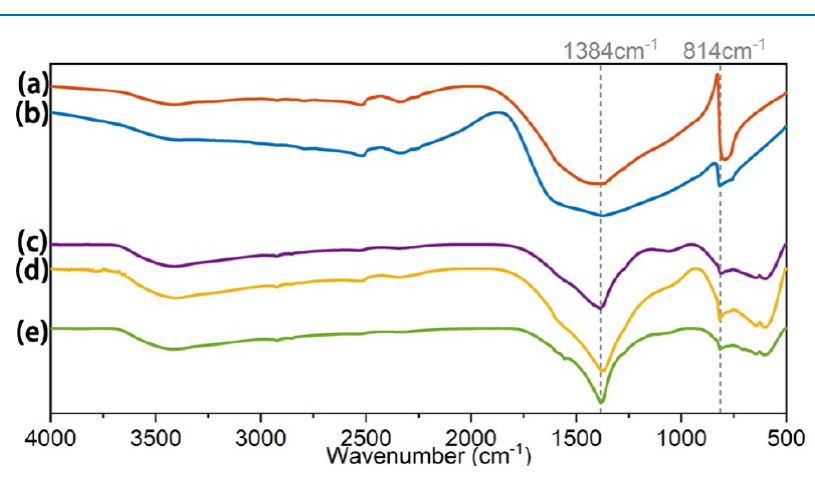

Figure 7. Fourier translation infrared spectra of (a) submicrometer h$\mathrm{BN},(\mathrm{b})$ commercially available h-BN $(23 \mu \mathrm{m}$ D 50$)$, (c) BNNSs obtained from submicrometer h-BN, (d) BNNSs obtained from commercially available h-BN (23 $\mu \mathrm{m}$ D50), and (e) BNNSs obtained from submicrometer $\mathrm{h}-\mathrm{BN}$ with additives.

after ball milling are quite different, indicating that the submicrometer h-BN and commercially available h-BN were effectively exfoliated. The peak at $814 \mathrm{~cm}^{-1}$ can be seen in the figure as deformation vibration of the $\mathrm{B}-\mathrm{N}$ bond. The peak at $1384 \mathrm{~cm}^{-1}$ indicates the stretching vibration of the $\mathrm{B}-\mathrm{N}$ bond, which is more obvious for BNNSs than h-BN.

3.2.4. Characterization of Purity of BNNSs. SEM and EDS analyses of BNNSs obtained by the ball milling process from submicrometer h-BN are shown in Figure 8a,b, from which it can be seen that $\mathrm{B}, \mathrm{N}$, and $\mathrm{O}$ were detected and uniformly distributed in the sample. Combined with the XPS analysis 

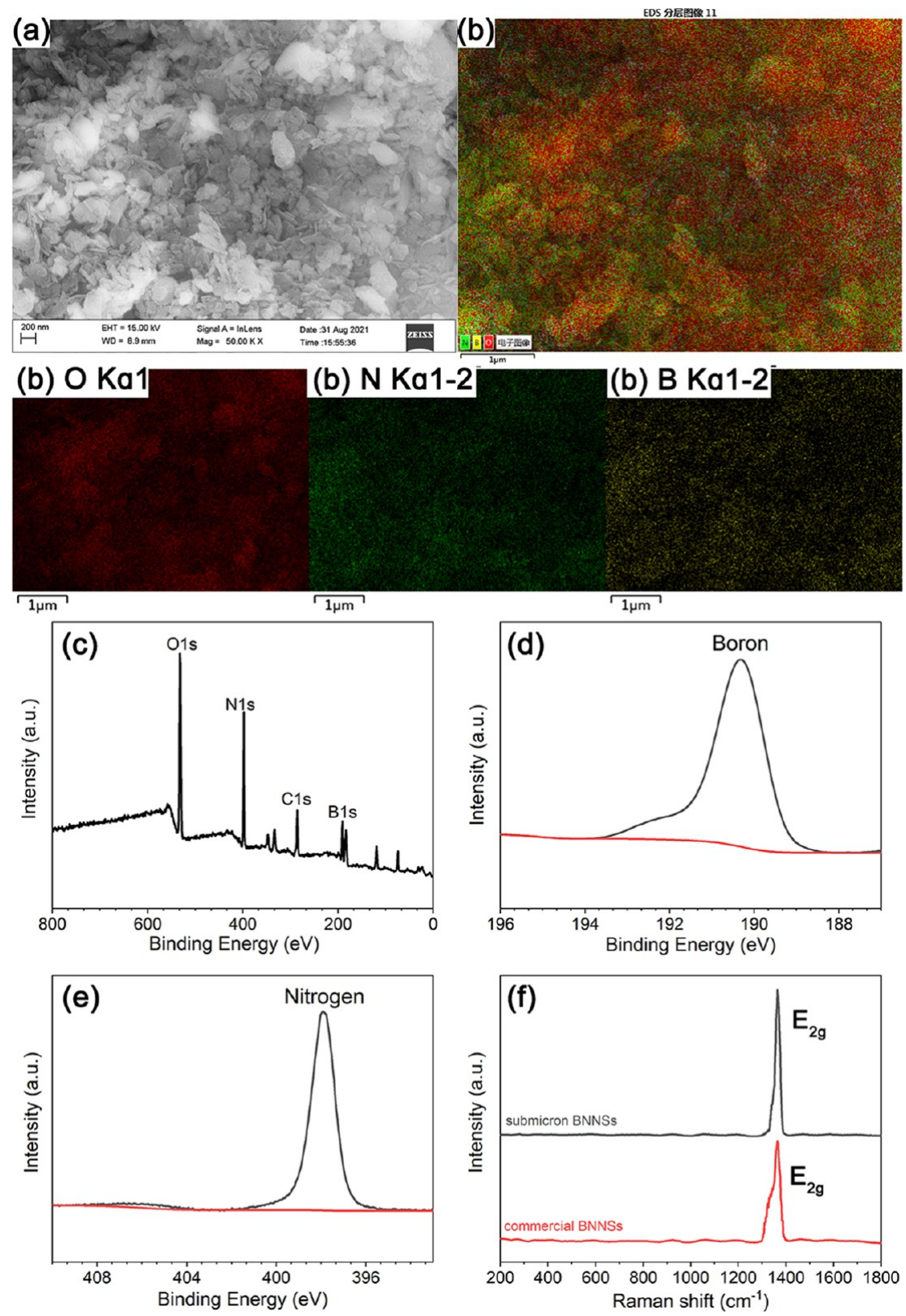

Figure 8. (a, b) SEM and corresponding elemental distribution diagrams (EDS) of BNNSs obtained from submicrometer h-BN through the ball milling process; (c-e) XPS of BNNSs obtained through the ball milling process of submicrometer h-BN; (f) the Raman spectra of BNNSs obtained by ball milling of submicrometer h-BN and commercially available h-BN (23 $\mu \mathrm{m}$ D50).
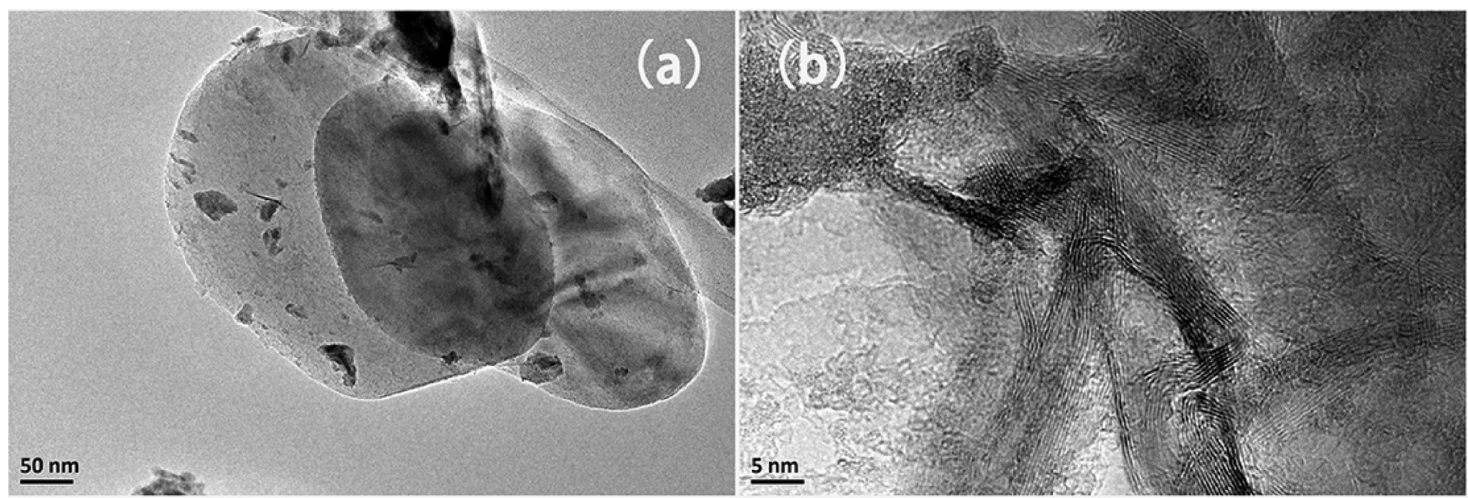

Figure 9. TEM images of BNNSs obtained from submicrometer-scale h-BN after ball milling with additives under magnifications of (a) 5000 times and (b) 50,000 times. 
shown in Figure 8c-e, the synthesis of BNNSs is successful. As can be seen in the Raman spectra of the two kinds of obtained BNNSs in Figure 8f, the peak at $1360 \mathrm{~cm}^{-1}$ is the specific vibrational mode $(E 2 g)$ of h-BN, which was caused by the plane vibration of the sp2 hybridized $\mathrm{B}-\mathrm{N}=\mathrm{B}$ bond. The more intense and narrow peak indicates less defect structures. Clearly, BNNSs obtained from submicrometer h-BN have a better structure than those obtained from commercially available hBN.

3.2.5. Effects of Ball Milling Additives on the BNNS Structure. Adding different kinds of additives during the ball milling process, such as hydrophilic and lipophilic amine-based molecules, affects the structure and size of BNNSs to some extent. Adding a certain amount of dopamine makes products appear off-white and hardly sedimentate within $72 \mathrm{~h}$ while the dispersion was homogeneous and stable. Therefore, due to the insertion of dopamine molecules in the h-BN interlayer during ball milling promoting the exfoliation process, the addition of an appropriate amount of ball milling additives would contribute to the effective exfoliation of $\mathrm{h}-\mathrm{BN}$ to a certain level. However, the existence of dopamine can also bring impurities into the product, which increases the difficulty of separation and washing of subsequent products. As shown in Figure 9, the obtained BNNSs appear to be transparent and more evenly distributed. However, prisms of the edge of nanosheets become smaller and most of them appear circular. Thickness is about 2 to $10 \mathrm{~nm}$ and the number of BNNS layers ranges from 3 to 15 , which indicates that the atomic interaction between amine molecules and the edge structure of h-BN promotes the effective exfoliation of hBN.

The AFM photographs and height characterization of BNNSs deposited on the silicon chip are shown in Figure 10. It can be seen that BNNSs obtained from commercially available h-BN (23 $\mu \mathrm{m}$ D50) have larger thickness, while BNNSs obtained from submicrometer h-BN show smaller thickness $(\leq 5 \mathrm{~nm})$ and less layer structures. This result once again confirms that the

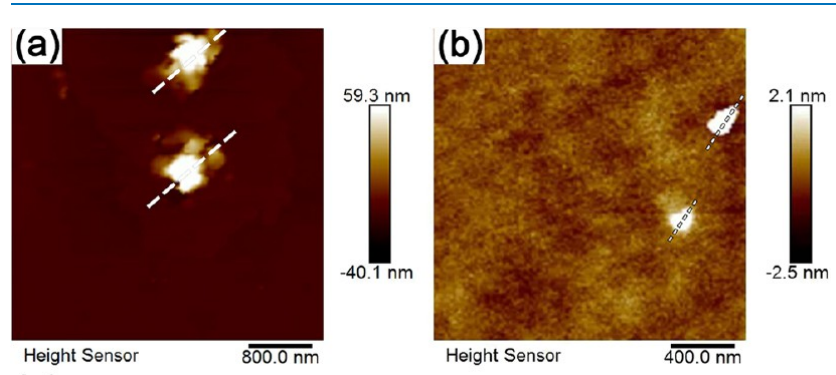

(a)
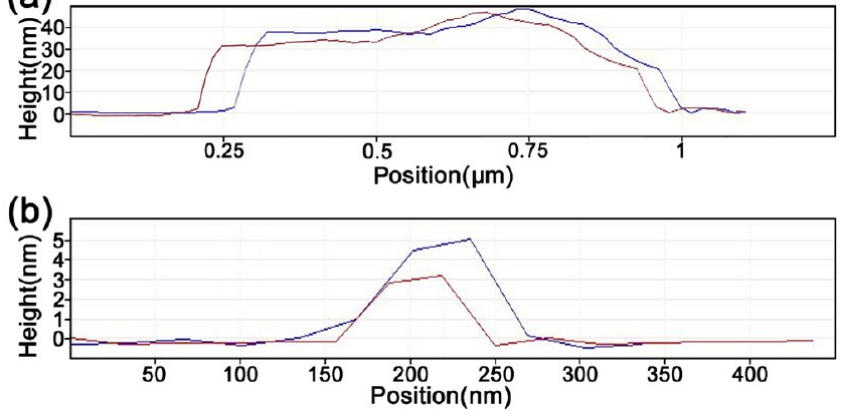

Figure 10. AFM and height images of BNNSs obtained from (a) commercially available h-BN (23 $\mu \mathrm{m}$ D50) and (b) submicrometer hBN. submicrometer h-BN prepared by this method is better than commercially available h-BN (23 $\mu \mathrm{m}$ D50).

\section{CONCLUSIONS}

Submicrometer-sized h-BN was first synthesized by the hightemperature solid-phase method. The reaction conditions that include reaction temperature, reaction medium, and reaction time have a large effect on the structure and morphology of h$\mathrm{BN}$. With the reaction temperature ranging from 900 to $1200{ }^{\circ} \mathrm{C}$ and the reaction time ranging from 8 to $10 \mathrm{~h}$, the synthesized h$\mathrm{BN}$ particles were uniform with high crystallinity and submicrometer-scale particle size. Then, submicrometer-scale h-BN was used as a ball milling raw material to be effectively exfoliated by the wet ball milling technique. The results of this study showed that the BNNSs with uniform size distribution, which possesses advantages of a fairly high yield of $73 \%$, simple postprocessing, and less pollution, can be obtained by appropriately adjusting factors such as the ball-to-material ratio and ball milling additives.

\section{ASSOCIATED CONTENT}

\section{(3) Supporting Information}

The Supporting Information is available free of charge at https://pubs.acs.org/doi/10.1021/acsomega.1c03406.

Comparison of yield of BNNSs and cost of BNNS product (PDF)

\section{AUTHOR INFORMATION}

\section{Corresponding Author}

Benxue Zou - Department of Chemical Engineering, Eastern Liaoning University, Dandong 118001, China; 이이.org/ 0000-0003-0704-5036; Email: zoubenxue@elnu.edu.cn

\section{Authors}

Shaocheng Li - Department of Chemical Engineering, Eastern Liaoning University, Dandong 118001, China

Xianlang Lu - Department of Chemical Engineering, Eastern Liaoning University, Dandong 118001, China

Yanda Lou - Department of Chemical Engineering, Eastern Liaoning University, Dandong 118001, China

Kejun Liu - Department of Chemical Engineering, Eastern Liaoning University, Dandong 118001, China

Complete contact information is available at:

https://pubs.acs.org/10.1021/acsomega.1c03406

\section{Notes}

The authors declare no competing financial interest.

\section{ACKNOWLEDGMENTS}

This work was supported by the National Natural Science Foundation of China (51873084).

\section{REFERENCES}

(1) de los Reyes, C. A.; Hernández, K.; Martínez-Jiménez, C.; Walz Mitra, K. L.; Ginestra, C.; Smith McWilliams, A. D.; Pasquali, M.; Martí, A. A. Tunable Alkylation of White Graphene (Hexagonal Boron Nitride) Using Reductive Conditions. J. Phys. Chem. C 2019, 123, 19725-19733.

(2) Yu, C.; Zhang, J.; Tian, W.; Fan, X.; Yao, Y. Polymer composites based on hexagonal boron nitride and their application in thermally conductive composites. RSC Adv. 2018, 8, 21948-21967.

(3) Jedrzejczak-Silicka, M.; Trukawka, M.; Dudziak, M.; Piotrowska, K.; Mijowska, E. Hexagonal Boron Nitride Functionalized with Au 
Nanoparticles-Properties and Potential Biological Applications. Nanomaterials 2018, 8, 605.

(4) He, L.; Zeng, J.; Huang, Y.; Yang, X.; Li, D.; Chen, Y.; Yang, X.; Wang, D.; Zhang, Y.; Fu, Z. Enhanced Thermal Conductivity and Dielectric Properties of h-BN/LDPE Composites. Materials 2020, 13, 4738.

(5) Wan, D.; Wu, X.; Zhang, W.; Peng, K. Thermal conductivity and thermal expansion of few-layer h-BN/Cu composites. Mater. Res. Bull. 2019, 120, 110606.

(6) Fan, D.; Lv, X.; Feng, J.; Zhang, S.; Xie, J.; Liu, J. Integrating CoNi nanoparticles encapsulated by few-layer h-BN with excellent thermal conductivity and thermal stability. J. Alloys Compd. 2017, 704, 701706.

(7) Randhawa, K. S.; Patel, A. D. Enhancing tribo-mechanical properties and thermal stability of nylon 6 by hexagonal boron nitride fillers. E-Polymers 2020, 20, 733-745.

(8) Kleinerman, O.; Adnan, M.; Marincel, D. M.; Anson, W. K.; Ma, A. W.; Bengio, E. A.; Park, C.; Chu, S. H.; Pasquali, M.; Talmon, Y. Dissolution and Characterization of Boron Nitride Nanotubes in Superacid. Langmuir 2017, 33, 14340-14346.

(9) Hu, Y.; Yin, Y.; Ding, G.; Liu, J.; Zhou, H.; Feng, W.; Zhang, G.; Li, D. High thermal conductivity in covalently bonded bi-layer honeycomb boron arsenide. Mater. Today Phys. 2021, 17, 100346.

(10) Ahmadi, M. T.; Razmdideh, A.; Rahimian Koloor, S. S.; Petrů, M. Carbon-Based Band Gap Engineering in the h-BN Analytical Modeling. Materials 2020, 13, 1026.

(11) Kim, M.; Moon, S. W.; Kim, G.; Yoon, S. I.; Kim, K.; Min, S. K.; Shin, H. S. Effect of Pt Crystal Surface on Hydrogenation of Monolayer h-BN and Its Conversion to Graphene. Chem. Mater. 2020, 32, 45844590

(12) Zhang, Z.; Duan, X.; Qiu, B.; Chen, L.; Cai, D.; He, P.; Yang, Z.; Jia, D.; Zhou, Y. Improvement of grain size and crystallization degree of LPSed h-BN composite ceramics by amorphization/nanocrystallization of raw h-BN powders. J. Alloys Compd. 2021, 852, 156765.

(13) Wu, L.; Luo, N.; Xie, Z.; Liu, Y.; Chen, F.; Fu, Q. Improved breakdown strength of Poly(vinylidene Fluoride)-based composites by using all ball-milled hexagonal boron nitride sheets without centrifugation. Compos. Sci. Technol. 2020, 190, 108046.

(14) Tozar, A.; Karahan, İ. H.; Yücel, Y. Optimization of the Electrophoretic Deposition Parameters for Biocomposite Hydroxyapatite/Chitosan/Collagen/h-BN Coatings on $\mathrm{Ti}_{6} \mathrm{Al}_{4} \mathrm{~V}$ Biomedical Implants. Metall. Mater. Trans. A 2019, 50, 1009-1020.

(15) Ouyang, H.; Li, W.; Long, Y. Carbon-doped h-BN for the enhanced electrochemical determination of dopamine. Electrochim. Acta 2021, 369, 137682.

(16) Aal, S. A.; Alfuhaidi, A. K. Enhancement of hydrogen storage capacities of $\mathrm{Co}$ and $\mathrm{Pt}$ functionalized h-BN nanosheet: Theoretical study. Vacuum 2021, 183, 109838.

(17) Chen, T.-A.; Chuu, C.-P.; Tseng, C.-C.; Wen, C.-K.; Philip, H.S.; Pan, S.; Li, R.; Chao, T.-A.; Chueh, W.-C.; Zhang, Y.; Fu, Q.; Yakobson, B. I.; Chang, W.-H.; Li, L.-J. Wafer-scale single-crystal hexagonal boron nitride monolayers on $\mathrm{Cu}(111)$. Nature 2020, 579, 219-223.

(18) Sun, W.; Meng, Y.; Fu, Q.; Wang, F.; Wang, G.; Gao, W.; Huang, X.; Lu, F. High-Yield Production of Boron Nitride Nanosheets and Its Uses as a Catalyst Support for Hydrogenation of Nitroaromatics. ACS Appl. Mater. Interfaces 2016, 8, 9881.

(19) Hu, X.; Liu, J.; He, Q.; Meng, Y.; Cao, L.; Sun, Y.-P.; Chen, J.; Lu, F. Aqueous compatible boron nitride nanosheets for high-performance hydrogels. Nanoscale 2016, 8, 4260.

(20) Gonzalez-Ortiz, D.; Pochat-Bohatier, C.; Gassara, S.; Cambedouzou, J.; Bechelany, M.; Miele, P. Development of novel hBNNS/PVA porous membranes via Pickering emulsion templating. Green Chem. 2018, 20, 4319-4329.

(21) Biscarat, J.; Bechelany, M.; Pochat-Bohatier, C.; Miele, P. Graphene-like BN/gelatin nanobiocomposites for gas barrier applications. Nanoscale 2015, 7, 613-618.

(22) Hwang, G.; Kwon, Y.; Lee, J.; Jeong, Y. Enhanced mechanical and anisotropic thermal conductive properties of polyimide nanocomposite films reinforced with hexagonal boron nitride nanosheets. J. Appl. Polym. Sci. 2020, 138, 50324

(23) Zheng, L.; Liu, Z.; Liu, D.; Wang, X.; Li, Y.; Jiang, M.; Lin, F.; Zhang, H.; Shen, B.; Zhu, X.; Gong, Y.; Fang, Z. Deep subwavelength control of valley polarized cathodoluminescence in h-BN/WSe ${ }_{2} / \mathrm{h}-\mathrm{BN}$ heterostructure. Nat. Commun. 2021, 12, 291.

(24) Liu, H.; Su, X.; Fu, R.; Wu, B.; Chen, X. The flexible film of SCF/ BN/PDMS composites with high thermal conductivity and electrical insulation. Compos. Commun. 2021, 23, 100573.

(25) Chen, F.; Li, Z.; Zou, L.-F.; Ma, W.-J.; Li, J.-W.; Chen, Z.; Niu, Z.B.; Liu, P.-F.; Xiao, P. Tribological behavior and mechanism of h-BN modified copper metal matrix composites paired with C/C-SiC. Tribol. Int. 2021, 153, 106561 .

(26) Zhang, C.; Gao, B.; Ran, Y.; Shi, Z.; Zhu, H.; Zhang, H.; Liu, J.; Yang, B.; Liu, Z.; Wu, T.; Xie, X. Silicon-Assisted Growth of Hexagonal Boron Nitride to Improve Oxidation Resistance of Germanium. $2 D$ Mater. 2021, 8, No. 035041.

(27) Xie, Z.; Wu, K.; Liu, D.; Zhang, Q.; Fu, Q. One-Step AlkylModification on Boron Nitride Nanosheets for Polypropylene Nanocomposites with Enhanced Thermal Conductivity and UltraLow Dielectric Loss. Compos. Sci. Technol. 2021, 208, 108756.

(28) Qiu, J.; Wu, T.; Qu, J. Fabrication of iron oxide nanoparticle decorated boron nitride nanosheet for flame-retarding silicone rubber. Mater. Lett. 2021, 283, 128712.

(29) Sajid, A.; Ford, M. J.; Reimers, J. R. Single-photon emitters in hexagonal boron nitride: a review of progress. Rep. Prog. Phys. 2020, 83, No. 044501.

(30) Abbas, S.; Abbas, A.; Liu, Z.; Tang, C. The two-dimensional boron nitride hierarchical nanostructures: Controllable synthesis and superhydrophobicity. Mater. Chem. Phys. 2020, 240, 122145.

(31) Kim, J. H.; Pham, T. V.; Hwang, J. H.; Kim, C. S.; Kim, M. J. Boron nitride nanotubes: synthesis and applications. Nano Converg. 2018, 5, 17.

(32) Wang, X.; Yang, Y.; Jiang, G.; Yuan, Z.; Yuan, S. A facile synthesis of boron nitride nanosheets and their potential application in dye adsorption. Diamond Relat. Mater. 2018, 81, 89-95.

(33) Yang, N.; Ji, H.; Jiang, X.; Qu, X.; Zhang, X.; Zhang, Y.; Liu, B. Preparation of Boron Nitride Nanoplatelets via Amino Acid Assisted Ball Milling: Towards Thermal Conductivity Application. Nanomaterials 2020, 10, 1652.

(34) Gonzalez-Ortiz, D.; Salameh, C.; Bechelany, M.; Miele, P. Nanostructured boron nitride-based materials: synthesis and applications. Mater. Today Adv. 2020, 8, 100107.

(35) Li, Y.; Zhang, W.; Guo, B.; Datta, D. Interlayer shear of nanomaterials: Graphene-graphene, boron nitride-boron nitride and graphene-boron nitride. Acta Mech. Solida Sin. 2017, 30, 234-240.

(36) Han, R.; Khan, M. H.; Angeloski, A.; Casillas, G.; Yoon, C. W.; Sun, X.; Huang, Z. Hexagonal Boron Nitride Nanosheets Grown via Chemical Vapor Deposition for Silver Protection. Acs Appl. Nano Mater. 2019, 2, 2830-2835.

(37) Ikram, M.; Jahan, I.; Haider, A.; Hassan, J.; Ul-Hamid, A.; Imran, M.; Haider, J.; Shahzadi, A.; Shahbaz, A.; Ali, S. Bactericidal behavior of chemically exfoliated boron nitride nanosheets doped with zirconium. Appl. Nanosci. 2020, 10, 2339-2349.

(38) Tian, Z.; Chen, K.; Sun, S.; Zhang, J.; Cui, W.; Xie, Z.; Liu, G. Crystalline boron nitride nanosheets by sonication-assisted hydrothermal exfoliation. J. Adv. Ceram. 2019, 8, 72-78.

(39) Kumar, R.; Singh, R. K.; Yadav, S. K.; Savu, R.; Moshkalev, S. A. Mechanical pressure induced chemical cutting of boron nitride sheets into boron nitride quantum dots and optical properties. J. Alloys Compd. 2016, 683, 38-45.

(40) Chen, X.; Tan, C.; Liu, X.; Luan, K.; Guan, Y.; Liu, X.; Zhao, J.; Hou, L.; Gao, Y.; Chen, Z. Growth of hexagonal boron nitride films on silicon substrates by low-pressure chemical vapor deposition. J. Mater. Sci-Mater. Electron. 2021, 32, 3713-3719.

(41) Ikram, M.; Hussain, I.; Hassan, J.; Haider, A.; Imran, M.; Aqeel, M.; Ul-Hamid, A.; Ali, S. Evaluation of antibacterial and catalytic potential of copper-doped chemically exfoliated boron nitride nanosheets. Ceram. Int. 2020, 46, 21073-21083. 
(42) Yang, G.; Wu, Y.; Zhu, B.; Li, J.; Fu, Y.; Gao, D.; Zhao, J.; Ma, S. Defect-related high temperature ferromagnetism in mechanically milled hexagonal boron nitride nanoplates. Appl. Surf. Sci. 2019, 487, 825-832.

(43) Gonzalez Ortiz, D.; Pochat-Bohatier, C.; Cambedouzou, J.; Bechelany, M.; Miele, P. Exfoliation of Hexagonal Boron Nitride (h$\mathrm{BN}$ ) in Liquide Phase by Ion Intercalation. Nanomaterials 2018, 8, 716.

(44) Bi, Y.-S.; Liu, B.; Liu, X.-Y.; Qin, Y.; Zou, B.-X. A h-BCN for Electrochemical Sensor of Dopamine and Uric Acid. J. Nanomater. 2020, 2020, 1-9. 